\title{
SARS-CoV-2 Persistence in Immunocompromised Children
}

\author{
Susan Dolan ${ }^{1}$, Jean Mulcahy Levy ${ }^{2}$, Angla Moss ${ }^{2}$, Kelly Pearce ${ }^{1}$, Samuel Dominguez ${ }^{1}$, \\ Sarah Jung ${ }^{1}$, Molly Butler ${ }^{1}$, Eric Mwangi ${ }^{1}$, Kelly Maloney ${ }^{3}$, and Suchitra Rao ${ }^{2}$ \\ ${ }^{1}$ Childrenś Hospital Colorado \\ ${ }^{2}$ University of Colorado Denver School of Medicine \\ ${ }^{3}$ Children's Hospital Colorado
}

June 23, 2021

\begin{abstract}
Introduction/Objectives: We evaluated the length of time immunocompromised children (ICC) remain positive for SARSCoV-2, identified factors associated with viral persistence and determined cycle threshold (CT) values of children with viral persistence as a surrogate of viral load. Methods: We conducted a retrospective cohort study of ICC at a pediatric hospital from March 2020-2021. Immunocompromised status was defined as primary, secondary or acquired due to medical comorbidities/immunosuppressive treatment. The primary outcome was time to first-of-two consecutive negative SARS-CoV-2 Polymerase chain reaction (PCR) tests at least 24 hours apart. Testing of sequential clinical specimens from the same subject was conducted using the Centers for Disease Control (CDC) 2019-nCoV Real-Time RT-PCR Diagnostic Panel assay. Descriptive statistics, Kaplan-Meier curve median event times and log-rank-sum tests were used to compare outcomes between groups. Results: Ninety-one children met inclusion criteria. Median age was 15.5 years (IQR 8-18 yrs), $64 \%$ were male, $58 \%$ were white, and $43 \%$ were Hispanic/Latinx. Most (67\%) were tested in outpatient settings and $58 \%$ were asymptomatic. The median time to two negative tests was 42 days (IQR 25.0,55.0), with no differences in median time by illness presentation or level of immunosuppression. Seven children had $>1$ sample available for repeat testing, and 5/7 (71\%) children had initial CT values of $<30$, (moderate to high viral load); 4 children had CT values of $<303-4$ weeks later, suggesting persistent moderate to high viral loads. Conclusions: Most ICC with SARS-CoV-2 infection had mild disease, with prolonged viral persistence $>6$ weeks and moderate to high viral load.
\end{abstract}

\section{SARS-CoV-2 Persistence in Immunocompromised Children}

Authors: Susan A. Dolan, Jean Mulcahy Levy, Angela Moss, Kelly Maloney, Kelly Pearce, Sarah Jung, Samuel R. Dominguez, Molly Butler, Eric Mwangi, Suchitra Rao

\section{Affiliations:}

Susan A. Dolan, MSN, RN, CIC FAPIC; Department of Epidemiology, Children's Hospital Colorado, Aurora, CO, USA

Jean Mulcahy Levy, MD; Department of Pediatrics (Center for Cancer and Blood Disorders), University of Colorado School of Medicine and Children's Hospital Colorado, Aurora, CO, USA Morgan Adams Foundation Pediatric Brain Tumor Research Program, Children's Hospital Colorado, Aurora, United States Department of Pharmacology, University of Colorado School of Medicine, Aurora, CO 80045, USA

Angela Moss, MS; University of Colorado School of Medicine, Children's Hospital Colorado and Adult and Child Center for Health Outcomes Research and Delivery Science, Aurora, CO, USA

Kelly W. Maloney, MD; Department of Pediatrics (Center for Cancer and Blood Disorders) and Children's Hospital Colorado, Aurora, CO, USA 
Kelly Pearce, BA; Department of Epidemiology, Children's Hospital Colorado, Aurora, CO, USA

Sarah Jung, PhD; Department of Pathology and Laboratory Medicine, Children's Hospital Colorado, Aurora, CO, USA

Samuel R. Dominguez, MD, PhD; Department of Pediatrics (Infectious Diseases) University of Colorado School of Medicine and Children's Hospital Colorado, Aurora, CO, USA

Molly Butler, PhD; Department of Pathology and Laboratory Medicine, Children's Hospital Colorado, Aurora, CO, USA

Eric Mwangi, BS; Department of Pathology and Laboratory Medicine, Children's Hospital Colorado, Aurora, CO, USA

Suchitra Rao, MBBS, MSCS; Department of Pediatrics (Infectious Diseases and Hospital Medicine), University of Colorado School of Medicine and Children's Hospital Colorado, Aurora, CO, USA

Short Running Title: SARS-CoV-2 Persistence in Immunocompromised Children

Keywords: SARS CoV-2, COVID-19, shedding, viral persistence, immunocompromised, pediatrics

Corresponding Author: Suchitra Rao, MBBS, MSCS, Department of Pediatrics (Infectious Diseases, Hospital Medicine and Epidemiology), University of Colorado School of Medicine and Children's Hospital Colorado, Box 055, 13123 E. 16th Ave, Aurora, CO 80045, USA; phone: (720) 777 2823; fax: 7207777295 email: suchitra.rao@childrenscolorado.org.

Funding Source: none

Conflicts of interest: Suchitra Ro All other authors have no conflicts of interest to disclose.

\section{Abbreviations:}

\begin{tabular}{ll}
\hline Abbreviation & Full Term \\
\hline ALC & Absolute lymphocyte count \\
ANC & Absolute neutrophil count \\
CAR-T & Chimeric antigen receptor T cell therapy \\
CCBD & Center for Cancer and Blood Disorders \\
CCBD & Center for Cancer and Blood Disorders \\
COVID-19 & Coronavirus Disease 2019 \\
CT & Cycle threshold \\
HIV & Human immunodeficiency virus \\
HSCT & Hematopoietic Stem Cell Transplant \\
ICC & Immunocompromised children \\
ICU & Intensive care unit \\
IQR & Interquartile Range \\
KM & Kaplan-Meier \\
PCR & Polymerase chain reaction \\
RNA & Ribonucleic acid \\
RT & Reverse Transcriptase \\
SARS-CoV-2 & Severe Acute Respiratory Syndrome Coronavirus 2 \\
SOT & Solid organ tumor \\
\hline
\end{tabular}

Word Count abstract: 250

Word Count main text: 2742

Number of Tables and Figures: 7 


\section{Objectives:}

We evaluated the length of time immunocompromised children (ICC) remain positive for SARS-CoV-2, identified factors associated with viral persistence and determined cycle threshold $\left(\mathrm{C}_{\mathrm{T}}\right)$ values of children with viral persistence as a surrogate of viral load.

\section{Methods:}

We conducted a retrospective cohort study of ICC at a pediatric hospital from March 2020-2021. Immunocompromised status was defined as primary, secondary, or acquired due to medical comorbidities/immunosuppressive treatment. The primary outcome was time to first-of-two consecutive negative SARS-CoV-2 polymerase chain reaction (PCR) tests at least 24 hours apart. Testing of sequential clinical specimens from the same subject was conducted using the Centers for Disease Control (CDC) 2019-nCoV Real-Time RT-PCR Diagnostic Panel assay. Descriptive statistics, Kaplan-Meier curve median event times and log-rank tests were used to compare outcomes between groups. Results:

Ninety-one children met inclusion criteria. Median age was 15.5 years (IQR 8-18 yrs), 64\% were male, 58\% were white, and $43 \%$ were Hispanic/Latinx. Most $(67 \%)$ were tested in outpatient settings and $58 \%$ were asymptomatic. The median time to two negative tests was 42 days (IQR 25.0-55.0 days), with no differences in median time by illness presentation or level of immunosuppression. Seven children had $>1$ sample available for repeat testing, and 5/7 (71\%) children had initial $\mathrm{C}_{\mathrm{T}}$ values of $<30$, (moderate to high viral load); 4 children had $\mathrm{C}_{\mathrm{T}}$ values of $<303-4$ weeks later, suggesting persistent moderate to high viral loads.

\section{Conclusions:}

Most ICC with SARS-CoV-2 infection had mild disease, with prolonged viral persistence $>6$ weeks and moderate to high viral load.

\section{Introduction}

The Coronavirus Disease 2019 (COVID-19) pandemic caused by the Severe Acute Respiratory Syndrome Coronavirus 2 (SARS-CoV-2) virus continues to be an ongoing global health crisis. SARS-CoV-2 causes mild to moderate upper respiratory tract infection in most children, but the true burden of disease is unknown due to asymptomatic infection. Viral load typically peaks with symptom onset and falls to undetectable levels by the third week, when patients generally begin to develop antibodies. ${ }^{1}$

Patients with a suppressed immune system, such as those being treated for cancer, are susceptible to more severe viral infections. ${ }^{2}$ While these infections, including SARS-CoV-2, have potential to be more severe in immunocompromised adults ${ }^{3,4}$, emerging data suggests that SARS-CoV-2 does not necessarily present with an increased risk of severe disease in immunocompromised children (ICC). ${ }^{5-8}$ However, the persistence of SARS-CoV-2 infection in pediatric immunosuppressed patients is still unknown, with case series suggesting that infection can be more prolonged in this population. ${ }^{9}$

An understanding of viral persistence in immunocompromised hosts has important public health implications. Specific impacts include the effective prevention of disease transmission through infection control practices, as well as decreasing the risk of emerging variants given the concern that SARS-CoV-2 variants may have emerged from prolonged replication within immunocompromised hosts. ${ }^{10,11}$ The objectives of our study were to determine the length of time that ICC are positive for SARS-CoV-2; identify the sociodemographic and clinical factors associated with prolonged viral shedding; and determine the $\mathrm{C}_{\mathrm{T}}$ values of a subset of patients with viral persistence as a surrogate of viral load and potential transmissibility.

\section{Methods:}

\section{Study design}

We conducted a retrospective cohort study of children evaluated for SARS-CoV-2 at Children's Hospital Colorado (CHCO) from March 19, 2020 to March 28, 2021. We included children who were positive for 
SARS-CoV-2 by PCR and were concurrently confirmed as immunocompromised by study investigators via chart review.

Setting

$\mathrm{CHCO}$ is a regional, academic quaternary-care pediatric hospital with affiliated sites serving children in the Denver metropolitan area, the greater Colorado area, and the seven surrounding states. The main hospital campuses in Aurora and Colorado Springs, in conjunction with six satellite locations, have 560 beds and admits over 15,000 inpatients per year. The Center for Cancer and Blood Disorders (CCBD) provides clinical services for children, adolescents, and young adults with cancer and non-malignant blood disorders at Children's Hospital Colorado and Network of Care locations. Study investigators extracted data from the electronic health record via systematic, concurrent and retrospective chart review. Approval was obtained from the Colorado Multiple Institutional Review Board (COMIRB \# 20-1578).

\section{Participants}

We included symptomatic and asymptomatic children in both inpatient and ambulatory settings seeking care at $\mathrm{CHCO}$ who were immunocompromised and had at least 1 positive SARS-CoV-2 PCR conducted at the CHCO Clinical Microbiology Laboratory. Children were defined as immunocompromised if they were a patient of the CCBD receiving active treatment for cancer (solid and liquid tumor), post bone marrow/stem cell transplant or chimeric antigen receptor- (CAR) T cell therapy with active documented immunodeficiency, had a primary or secondary immunodeficiency (e.g. HIV) or received immunosuppressive medications. We excluded children with normal immune function and those not currently on treatment for their cancer diagnosis, fully immune reconstituted post-bone marrow transplant, and those with a positive SARS-CoV-2 test at outside facilities only. Our institution requires ICC to have two consecutive negative SARS-CoV-2 PCR tests collected at least 24 hours apart and 21 days from the patient's initial positive test in order to be removed from isolation.

\section{Testing}

Nasopharyngeal swabs, nasal aspirates, tracheal aspirates, and bronchoalveolar lavage (BAL) samples were tested for detection of SARS-CoV-2 in Clinical Microbiology Laboratory at CHCO. The molecular assays performed included the CDC 2019-nCoV Real-Time RT-PCR Diagnostic Panel, Abbott RealTime SARSCoV-2 assay (Abbott Laboratories, Chicago, IL), the Simplexa ${ }^{\circledR}$ COVID-19 Direct assay (DiaSorin Molecular LLC, Cypress, CA), the BioFire ${ }^{\circledR}$ Respiratory Panel 2.1 (BioFire Diagnostics, Salt Lake City, UT), and the Xpert ${ }^{\circledR}$ Xpress SARS-CoV-2/Flu/RSV assay (Cepheid, Sunnyvale, CA). Testing was completed per manufacturer instructions as outlined in the respective Emergency Use Authorization Instructions for use ${ }^{12-15}$. Residual specimens from a subset of study subjects with persistently positive SARS-CoV-2 were re-tested using the CDC 2019-nCoV Real-Time RT-PCR Diagnostic Panel. Reported $\mathrm{C}_{\mathrm{T}}$ values were calculated by taking the mean of the $\mathrm{C}_{\mathrm{T}}$ values for the $\mathrm{N} 1$ and $\mathrm{N} 2$ viral gene targets. For samples in which SARS-CoV-2 was not detected, a $\mathrm{C}_{\mathrm{T}}$ value of 40 was assigned.

Variables

Variables of interest included demographic (age, sex, race, ethnicity), clinical (immunocompromising condition, chemotherapy phase, test indication and location), laboratory data (absolute neutrophil count [ANC], absolute lymphocyte count [ALC] within 72 hours of initial test), microbiologic data (sources, dates and results of SARS -CoV-2 testing) and immunocompromising medication (name, class, therapy phase). We defined low ANC or ALC as a level $<1000$ cells/uL, and moderately low ANC or ALC as a level of 1000-1500 cells/uL.

\section{Outcomes}

The primary outcome was time in days to the first of two consecutively negative tests. Secondary outcomes included time to the first negative test, hospitalization related to SARS-CoV-2 infection and $\mathrm{C}_{\mathrm{T}}$ values from PCR assays. 


\section{Statistical methods}

For all study objectives, unless otherwise specified, we summarized data descriptively using frequencies for categorical variables and measures of central tendency for continuous variables. Proportions were compared using the Chi-square test or the Fisher exact test when needed. Mean values were compared using the Wilcoxon-Rank-Sum or Kruskal-Wallis test were used.

Bivariable analyses were performed to compare patient and clinical characteristics by immunocompromising condition and to compare factors with dichotomized version of time to negative test result $(<30$ days, $>30$ days). For those that had at least one subsequent test, multiple SARS-CoV-2 test results were described graphically using a line segment plot. Kaplan-Meier (KM) analysis was stratified by oncology classification, symptomatic/asymptomatic, and ALC classification. KM curves were described by median times to negative (defined as first of two consecutive negatives) and compared with log rank tests. For those with at least one subsequent test, subjects were considered censored at the last test if they did not have two consecutive negative test results.

\section{Results:}

\section{Participants/Descriptive Data:}

Excluding 983 of 1,078 SARS-CoV-2 positive children during our study period who were not considered immunocompromised, there were 91 children who met inclusion and exclusion criteria. The sociodemographic and clinical characteristics of children, classified as oncology patients versus those on other immunosuppressive agents, are shown in Table 1. The median age of children was 15.5 years (IQR 8-18 yrs), $64 \%$ were male, and $53 \%$ of children were white, with $43 \%$ being Hispanic or Latinx. Most children (67\%) were tested in outpatient settings, and $58 \%$ of children were asymptomatic at the time of their first positive test. A higher proportion of children with an oncologic diagnosis were male, but otherwise there were no significant differences in demographics, hospitalization or testing indication between children with oncologic diagnoses and children with other immunocompromising conditions.

The median number of repeat tests among children in our cohort was 2 (IQR 0,4). The number of SARSCoV-2 PCR tests and results for children in our cohort with more than 1 test are shown in Figure 1. Children with an oncologic diagnosis were more likely to have $>1$ SARS $-\mathrm{CoV}-2$ PCR test and complete blood count testing within 72 hours of their initial test. Children with an oncologic diagnosis were more likely to have a lower median ALC (550 vs 1015, p = 0.039) compared with children with other immunocompromising conditions.

\section{Outcome Data:}

The median time to the first of two consecutive negative tests in our cohort by KM analysis was 42 days (IQR 25.0-55.0), and 11 of the 68 children (16\%) had intermittent negative followed by positive results (Figure 1).

Children with an oncologic diagnosis had a longer median time to two consecutive negative tests compared with children receiving other immunocompromising medications (38.0 [IQR 25.0-55.0] vs 30.0 [IQR 21.044.0] days), but these findings were not statistically significant $(\mathrm{p}=0.097)$ (Table 1$)$. KM analysis which included the censored subjects also showed there was no difference in times to two consecutive negative tests between these groups (Figure 2, log rank $\mathrm{p}=0.88$ ). KM analyses demonstrated that children with leukemia and lymphoma had a longer time to negative testing (43 days, IQR 25,55), versus children with a solid tumor diagnosis (35 days [IQR 26,50], log rank $\mathrm{p}=0.33$ ) (Figure 3). Children with low ALC $(<1000$ cells/uL) had longer time to negative testing (44 days, IQR 25,55) days compared with those with a moderately low to normal ALC (31 [IQR 26-44] days), $\log$ rank $\mathrm{p}=0.17$. There was no statistically significant difference between time to negative testing for children with symptomatic vs asymptomatic illness (42 [IQR 30-55] vs 38 [IQR 22-55] days, $\log$ rank $\mathrm{p}=0.59)$.

Secondary outcome data/other analyses: 
We compared characteristics of children with a first negative test within 30 days of their initial SARS-CoV-2 diagnosis compared with children with a first negative test after 30 days (Table 2). Children who took $>30$ days for their SARS-CoV-2 tests to turn negative were more likely to be receiving chemotherapy and steroids, but these findings were not statistically significant. In our cohort, of the 30 children who were hospitalized, $23(77 \%)$ hospitalizations were related to COVID-19 illness, with 3 children requiring ICU admission. One patient died from ARDS. This patient was a 20 -year-old male day +127 post matched unrelated donor for treatment-induced leukemia. He presented to clinic with a known SARS-CoV-2 positive test, progressive shortness of breath, tachypnea, and baseline oxygen saturation in the 80-85\% range. The patient had worsening respiratory and cardiac function and progressed to positive pressure ventilation with intubation on day 12 of admission and extracorporeal membrane oxygenation (ECMO) on day 25 of admission. SARS-CoV-2 directed therapy for this patient included remdesivir (10-day course), prednisone, and anakinra in consultation with infectious diseases and rheumatology. Following further worsening lung and right heart function, the family elected to withdraw life-sustaining treatment on day 37 of admission.

$C_{T}$ values

Clinical SARS-CoV-2 testing was completed on five different platforms depending on patient encounter classification and turn-around-time requirements. Therefore, to standardize results and enable further characterization of SARS-CoV-2 $\mathrm{C}_{\mathrm{T}}$ values, we identified a subgroup of 7 children who had multiple respiratory samples available for repeat testing. Demographic and clinical information regarding this subgroup is summarized in Table 3.

Using the CDC assay for repeat testing, we evaluated SARS-CoV-2 $\mathrm{C}_{\mathrm{T}}$ values for a total of 20 specimens from these 7 individuals over time, presuming $\mathrm{C}_{\mathrm{T}}$ value as a surrogate for viral load. Five of $7(71 \%)$ children had initial $\mathrm{C}_{\mathrm{T}}$ values $<30$, indicating a moderate to high viral load, and of these, $4(57 \%)$ children had repeat testing 21 to 30 days later with $\mathrm{C}_{\mathrm{T}}$ values $<30$, suggesting persistence of moderate to high viral loads (Figure $3)$. Two of $7(29 \%)$ patients had a single negative test followed by a positive test. By 40 days after initial positive testing, 6 of $7(86 \%)$ children had negative tests (indicated as $\mathrm{C}_{\mathrm{T}}$ of 40 ) or $\mathrm{C}_{\mathrm{T}}$ values $>35$. The exception was one patient who remained positive until day 85. One patient (Patient 4) had a positive result on a specimen when tested clinically but a negative result when re-tested on the CDC assay. This is most likely indicative of a low viral load in the specimen, since the assay used for clinical testing and the CDC assay have similar, but not identical, limits of detection.

\section{Discussion:}

In our study evaluating SARS-CoV-2 viral persistence in ICC, we found that the median time to the first of two negative tests was 6 weeks. Most children were tested in outpatient settings prior to admission or for procedural sedation, and over half of those tested were asymptomatic at the time of their first positive test. There was no significant difference in time to negativity among symptomatic versus asymptomatic children. Among children who remained positive at day 30, half had $\mathrm{C}_{\mathrm{T}}$ values $<30$, suggesting persistence of moderate to high viral loads and assumed potential risk of transmission. Our study highlights the importance of PCR-based screening for ICC to guide isolation duration, irrespective of symptom presentation. These data support our current practice, with the need for at least two negative tests, given the potential for intermittent negative followed by positive results, and risk for ongoing, potential transmission even several weeks from initial infection.

The CDC recommends an isolation period of 10 days in healthy, non-immunocompromised individuals with SARS-CoV-2 infection based on studies that demonstrate the absence of viable/transmissible virus beyond this period. ${ }^{16}$ Immunocompromised patients can have a longer period of infectivity, but there is little available evidence in the literature to guide infection control practices. Our study revealed a prolonged period of viral persistence in ICC, with a longer period of positivity compared with a case series of ICC and a study of immunocompromised adults. ${ }^{9,17}$ Children with leukemia/lymphoma had longer viral persistence compared with children with other immunocompromising conditions, which we hypothesize is due to this population having the highest impairment of adaptive immunity. These children also represent the population of children 
with the greatest degree of lymphopenia, which has been shown in adults to be associated with more severe disease. ${ }^{18}$ Another study has also shown that patients critically ill from SARS-CoV-2 were more likely to have a decrease in CD3+CD4+ T-cells compared to non-critical patients. ${ }^{19}$ Additionally, a study of adult cancer patients found a general lack of T-cell response to $\mathrm{CoV} \mathrm{N}, \mathrm{M}$ and S proteins from SARS-CoV-2. While these findings suggested a lack of a protective T-cell response, the patients presented with mild illnesses despite relatively high viral loads. ${ }^{12}$ And although we had one death in our patient cohort post-bone marrow transplant, allogeneic, autologous and CAR-T therapy patients have overall favorable outcomes following SARS-CoV-2 infection. ${ }^{13}$

Similar to findings from adult studies, we did not see a relationship between clinical presentation and persistent positive SARS-CoV-2 status. An adult study of 3758 adults re-tested following initial SARS-CoV-2 infection found longer time to negativity to be associated with older age, multiple comorbidities and solid organ transplant but not by degree of immunocompromise or illness severity ${ }^{17}$. Our findings serve as a reminder that even asymptomatic children may have potentially transmissible virus several weeks after initial detection. Asymptomatic ICC are a potentially important group that pose high risk of transmissibility given their silent infection and prolonged infectious period.

While PCR is unable to differentiate actively replicating virus from viral shedding, $\mathrm{C}_{\mathrm{T}}$ values may serve as a useful surrogate for viral load and potential transmissibility. Other studies suggest that $\mathrm{C}_{\mathrm{T}}$ values of 25-30 represent moderate to high viral loads. We evaluated $\mathrm{C}_{\mathrm{T}}$ values using the same PCR assay for a small sample of patients with available clinical samples, which demonstrated that half still had a moderately high viral load even 3-4 weeks from their initial viral infection, regardless of their presentation. Other studies evaluating samples with PCR, viral culture, and subgenomic RNA have shown that $\mathrm{C}_{\mathrm{T}}$ values similar to those found in our study were associated with viable, culturable virus, as well as the presence of subgenomic RNA, which indicates actively replicating virus. ${ }^{20}$ These data further support the need for extended isolation duration for ICC given the persistence of relatively low $\mathrm{C}_{\mathrm{T}}$ values, suggesting potentially transmissible virus. Low CT values indicating relatively high viral loads were observed even in asymptomatic ICC, reflecting a need for test-based isolation protocols, rather than symptom-based isolation.

There are several limitations of this single-center, retrospective study. A small sample size precluded adjusted analyses to account for factors that may influence viral persistence and increased the risk of a type II error. Given the retrospective nature of the study, there was non-standardized repeat testing, and the frequency and intervals were not consistent between study participants. Repeat testing at our institution was recommended at 3 weeks from the first positive test, which may have increased our median time to negative testing, but most children in our cohort were still positive at this timepoint. We did not have a cohort of non-ICC with repeat SARS-CoV-2 testing, so we were not able to compare the degree of viral persistence in ICC with a healthy population. Finally, clinical SARS- $\backslash$ sout CoV-2 testing was conducted on different testing platforms, and there were a limited number of remaining sequential samples available for repeat testing to yield $\mathrm{C}_{\mathrm{T}}$ data.

In conclusion, our study demonstrates prolonged viral persistence of SARS-CoV-2 viral material in ICC, even in asymptomatic children, with moderate to high viral loads seen in the majority of children several weeks from initial positive testing. These findings highlight the importance of PCR-based screening for ICC to help guide isolation duration, irrespective of symptom presentation, given the risk for ongoing transmission even several weeks from initial infection.

Conflicts of Interest: Suchitra Rao and Samuel R. Dominguez received research support from BioFire Diagnostics; all other authors have no conflicts of interest to disclose.

Acknowledgements: We would like to acknowledge the Clinical Microbiology Laboratory at Children's Hospital Colorado for their tireless work during the pandemic.

\section{References:}

1. Sethuraman N, Jeremiah SS, Ryo A. Interpreting Diagnostic Tests for SARS-CoV-2. JAMA. 
2020;323(22):2249-2251.

2. Kaltsas A, Sepkowitz K. Community acquired respiratory and gastrointestinal viral infections: challenges in the immunocompromised host. Curr Opin Infect Dis. 2012;25(4):423-430.

3. Liang W, Guan W, Chen R, et al. Cancer patients in SARS-CoV-2 infection: a nationwide analysis in China. Lancet Oncol.2020;21(3):335-337.

4. Dai M, Liu D, Liu M, et al. Patients with Cancer Appear More Vulnerable to SARS-CoV-2: A Multicenter Study during the COVID-19 Outbreak. Cancer Discov. 2020;10(6):783-791.

5. Millen GC, Arnold R, Cazier JB, et al. Severity of COVID-19 in children with cancer: Report from the United Kingdom Paediatric Coronavirus Cancer Monitoring Project. Br J Cancer.2021;124(4):754-759.

6. Bisogno G, Provenzi M, Zama D, et al. Clinical Characteristics and Outcome of Severe Acute Respiratory Syndrome Coronavirus 2 Infection in Italian Pediatric Oncology Patients: A Study From the Infectious Diseases Working Group of the Associazione Italiana di Oncologia e Ematologia Pediatrica. J Pediatric Infect Dis Soc.2020;9(5):530-534.

7. Cleto-Yamane TL, Rodrigues-Santos G, de Magalhaes-Barbosa MC, et al. Screening of COVID-19 in outpatient children with cancer or solid organ transplantation: preliminary report. Eur J Pediatr. 2021.

8. Belsky JA, Tullius BP, Lamb MG, Sayegh R, Stanek JR, Auletta JJ. COVID-19 in immunocompromised patients: A systematic review of cancer, hematopoietic cell and solid organ transplant patients. $J$ Infect.2021;82(3):329-338.

9. El Dannan H, Al Hassani M, Ramsi M. Clinical course of COVID-19 among immunocompromised children: a clinical case series. BMJ Case Rep.2020;13(10).

10. Peacock TP, Penrice-Randal R, Hiscox JA, Barclay WS. SARS-CoV-2 one year on: evidence for ongoing viral adaptation. J Gen Virol.2021;102(4).

11. Kemp SA, Collier DA, Datir RP, et al. SARS-CoV-2 evolution during treatment of chronic infection. Nature. 2021;592(7853):277-282.

12. Mansi L, Spehner L, Daguindau E, et al. Study of the SARS-CoV-2-specific immune T-cell responses in COVID-19-positive cancer patients. Eur J Cancer. 2021;150:1-9.

13. Shah GL, DeWolf S, Lee YJ, et al. Favorable outcomes of COVID-19 in recipients of hematopoietic cell transplantation. J Clin Invest.2020;130(12):6656-6667.

14. U.S. Food and Drug Administration. (2020). Xpert Xpress SARS-CoV-2/Flu/RSV: Instructions for use https://www.fda.gov/media/142438/download. Accessed Accessed May 6, 2021.

15. U.S. Food and Drug Administration (2020). BioFire Respiratory Panel 2.1 (RP2.1): Instructions for use fda/gov/media/137583/download. Accessed Accessed May 6, 2021.

16. Perera R, Tso E, Tsang OTY, et al. SARS-CoV-2 Virus Culture and Subgenomic RNA for Respiratory Specimens from Patients with Mild Coronavirus Disease. Emerg Infect Dis. 2020;26(11):2701-2704.

17. Epstein RL SH, Hofman M, Lodi S, White LF, Barocas JA, Bouton TC, Xiao Y, et al. . Time to SARSCoV-2 PCR clearance in immunocompromising conditions: is test-based removal from isolation necessary in severely immunocompromised individuals? Open Forum Infectious Diseases.2021;ofab164.

18. Qin W, Bai W, Liu K, et al. Clinical Course and Risk Factors of Disease Deterioration in Critically Ill Patients with COVID-19. Hum Gene Ther. 2021;32(5-6):310-315.

19. Calvet J, Gratacos J, Amengual MJ, et al. CD4 and CD8 Lymphocyte Counts as Surrogate Early Markers for Progression in SARS-CoV-2 Pneumonia: A Prospective Study. Viruses. 2020;12(11). 
20. Truong TT, Ryutov A, Pandey U, et al. Increased viral variants in children and young adults with impaired humoral immunity and persistent SARS-CoV-2 infection: A consecutive case series. EBioMedicine.2021;67:103355.

\section{Hosted file}

TABLE 1.docx available at https://authorea.com/users/421499/articles/527507-sars-cov-2persistence-in-immunocompromised-children

\section{Hosted file}

Table 2 .docx available at https://authorea.com/users/421499/articles/527507-sars-cov-2persistence-in-immunocompromised-children

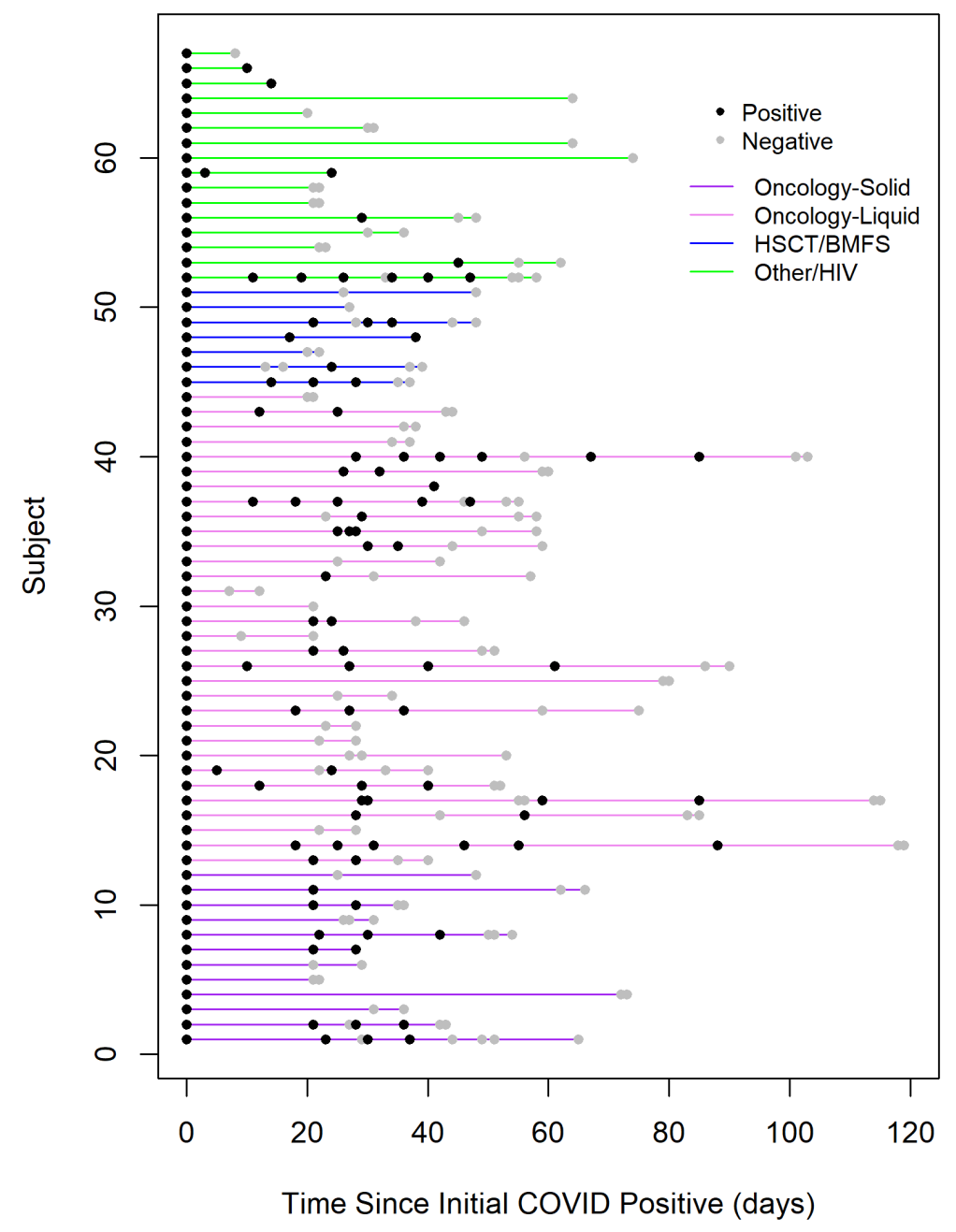



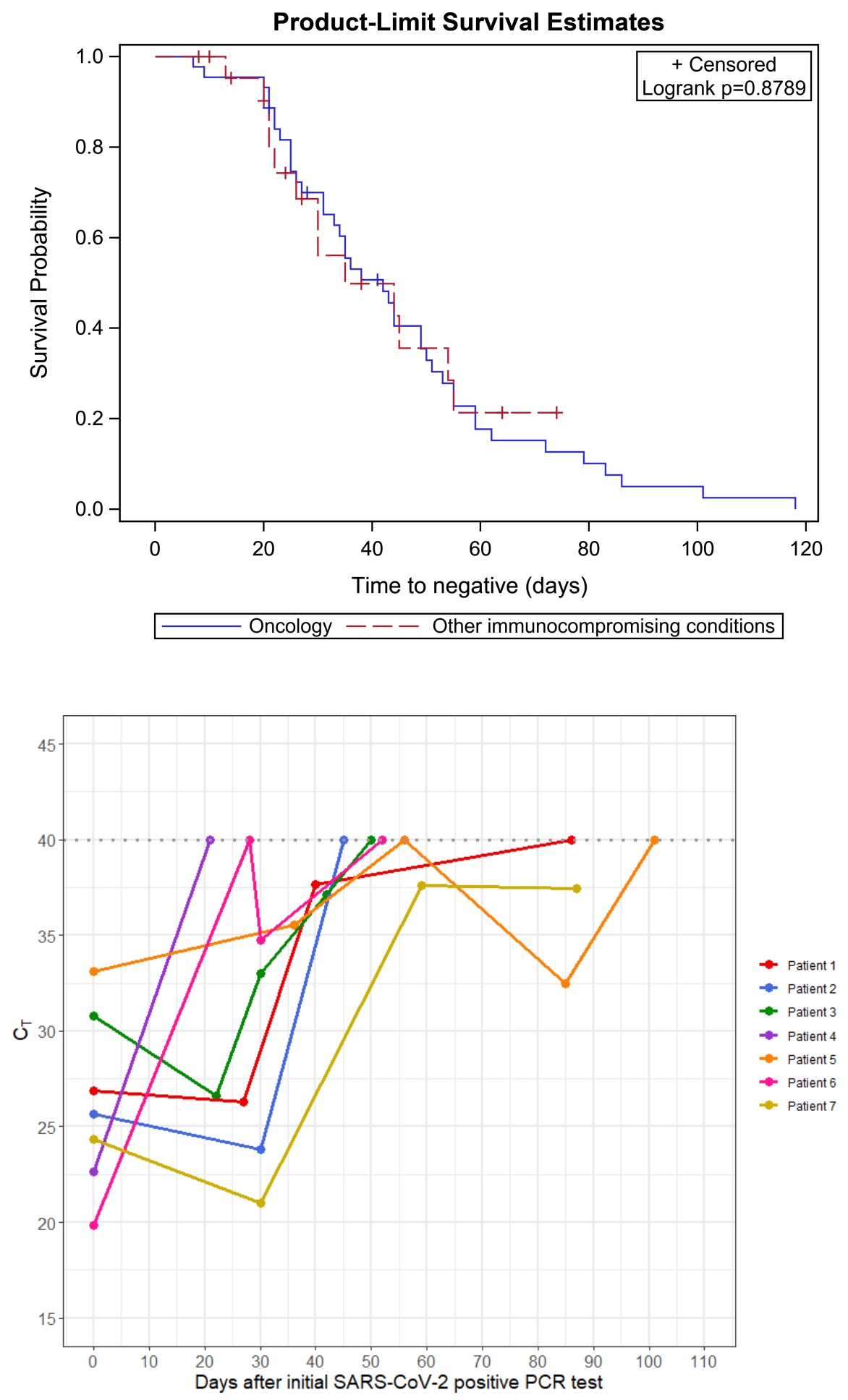